\title{
RESTRICCIÓN DE IMPORTACIONES EN TUNGURAHUA
}

ANA MARÍA SÁNCHEZ TOBAR ${ }^{1}$

JUAN PABLO MARTÍNEZ MESÍAS 2

\section{NEGOCIO}

\section{AUTOMOTOR}

En el intento de equilibrar la Balanza Comercial no petrolera del país a partir del año 2012, el Gobierno ha establecido ciertas medidas restrictivas a las importaciones, para varias partidas arancelarias, entre ellas, a la importación de vehículos. En este contexto el Ecuador cuenta con el Código Orgánico de la Producción, Comercio e Inversiones, el cual faculta al Comité de Comercio Exterior- COMEX en su parte pertinente a: "Regular, facilitar o restringir la exportación, importación, circulación y tránsito de mercancías no nacionales ni nacionalizadas, según los casos que estipule el Código y en los acuerdos internacionales debidamente ratificados por el Estado ecuatoriano" , otra de sus funciones es "Aprobar contingentes de importación o medidas restrictivas a las operaciones de comercio exterior (...)".

Las medidas más relevantes en cuanto a restricciones de importación se describen a continuación:

Figura 1. Cronología de medidas restrictivas de importación COMEX 2012 - 2016

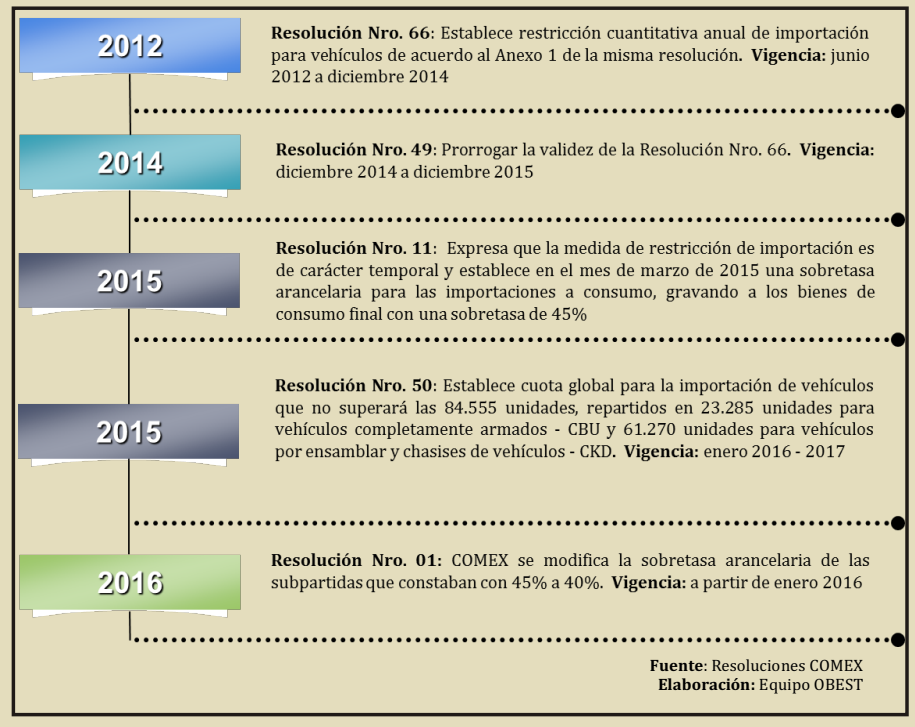

A pesar del cumplimiento del cronograma de desgravación propuesto por el Gobierno Nacional, las diferentes medidas restrictivas a la importación han limitado el crecimiento del sector automotor en el país ${ }^{3}$, Tungurahua no es la excepción, las ventas a partir de 2011 muestran tasas de decrecimiento a excepción de 2014 en donde crece 3\% para finalmente en 2015 presentar una drástica disminución de $33 \%$ en unidades, esto es 2.740 unidades menos de lo que se vendió en 2014. Similar comportamiento se registró en la provincia de Azuay, en donde también para el 2015 la reducción en unidades vendidas fue de $30 \%$, ambas provincias presentan una línea de tendencia a la baja como lo muestra la Figura 2.

${ }^{1}$ Economista - Especialista de Investigación y Desarrollo OBEST UTA

${ }^{2}$ Economista, Magíster en Administración de Empresas mención Planeación - Docente de la Facultad de Contabilidad y Auditoría e investigador del Observatorio Económico y Social de Tungurahua -UTA ${ }^{3}$ Ángela Meléndez Sánchez, El sector automotor, empeñado en su supervivencia, Revista GESTION, Julio 2013
Figura 2. Tasa de variación anual en ventas Tungurahua y Azuay 2008 - 2015

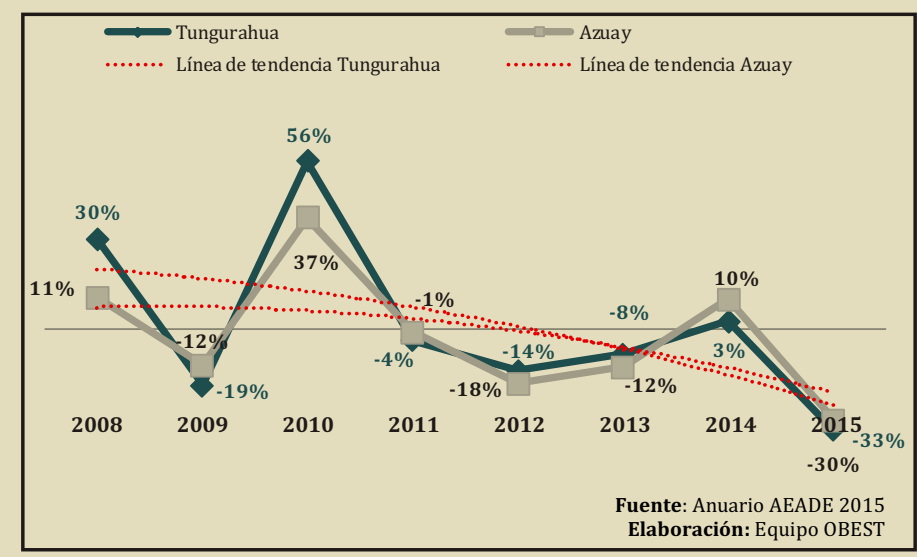

Las ventas de vehículos en la provincia de Tungurahua, muestran al segmento automóviles como el más representativo, abarcando el $34 \%$ de las ventas anuales en unidades, seguido del segmento camionetas con 27\%, a continuación SUV's y camiones con 19 y $13 \%$ respectivamente. Los segmentos que representan menor cantidad de unidades vendidas en la provincia son buses y VAN's con menos de $5 \%$ en cada uno.

En referencia a automóviles, en promedio anualmente se comercializan 2.739 unidades en Tungurahua, destacándose el año 2010 donde han alcanzado la mayor cantidad de venta de este segmento en los últimos nueve años, esto es, 3.701 unidades, mientras que en 2015 se muestra la menor cantidad de unidades con apenas 1.529 en el año, esto es el 41\% de lo vendido en 2010.

Figura 3. Porcentaje de venta de vehículos por segmento, Tungurahua 2007 - 2015

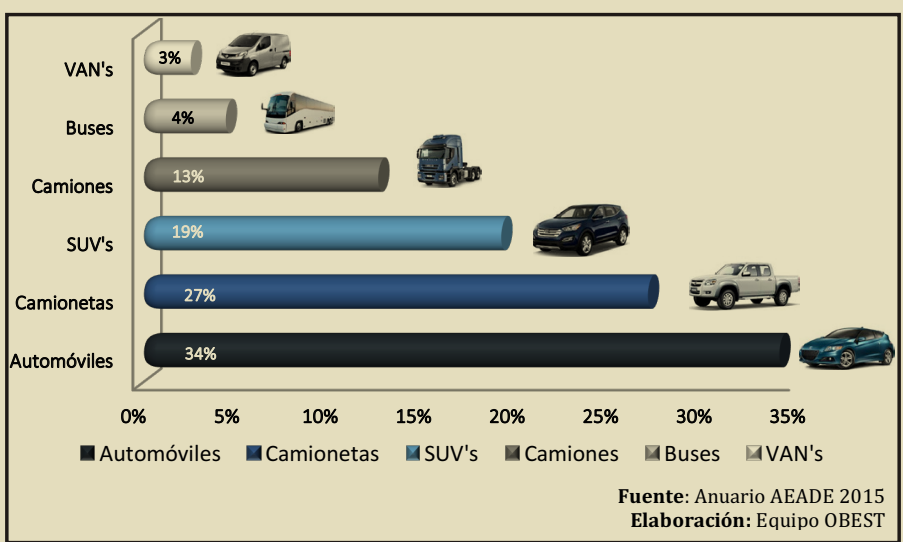

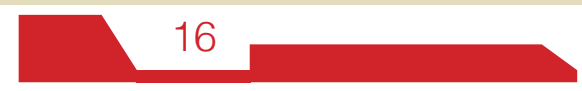


Los modelos más comercializados en los años 2014 y 2015 en Tungurahua corresponden a la marca CHEVROLET (detalle en la Tabla 1), comparando esta información con los resultados del primer sondeo de percepción a comerciantes de vehículos usados realizado por el Observatorio Económico y Social de Tungurahua OBEST, en donde los comerciantes manifestaron que más del $50 \%$ de las ventas que ejecutan corresponden a CHEVROLET también, se puede concluir que tanto para vehículos nuevos como usados la preferencia se inclina por la misma marca.

Tabla 1. Modelos de vehículos más vendidos en Tungurahua (unidades) 2014 - 2015

\begin{tabular}{|l|r|r|r|}
\hline \multicolumn{1}{|c|}{ MODELO } & 2014 & 2015 \\
\hline AK8JRSA HINO & 415 & 431 \\
\hline DMAX CHEVROLET & & & \\
\hline & & & \\
\hline
\end{tabular}

A partir del año 2011, la tendencia en ventas de vehículos en Tungurahua muestra decrecimientos constantes en las marcas $\mathrm{CHE}$ VROLET, KIA, HYUNDAI, FORD, RENAULT, NISSAN, MAZDA, TOYOTA, VOLKSWAGEN y CHERY, principalmente en 2015 en donde la reducción de ventas, en unidades promedio, es de 34\%, esto representa una disminución de 52\% de unidades entre el año 2011 y 2015. En el mercado de autos usados encuestado por el OBEST en febrero de 2016 , el $76 \%$ de comerciantes afirmó que las ventas habían disminuido en relación al año 2015, la tendencia en el mercado de autos nuevos no es diferente, como lo muestra la Figura 4. Sin embargo de este comportamiento, se observa un crecimiento considerable de la marca GREATWALL en donde a pesar de haber presentado una disminución de ventas en 2013, es la única marca que muestra crecimiento en el año 2015 en 27\%, vende 69 unidades más de las registradas en 2014.

UNIVERSIDAD TÉCNICA DE AMBATO
Figura 4. Evolución de variación anual de venta de vehículos por Marca Tungurahua 2010 - 2015

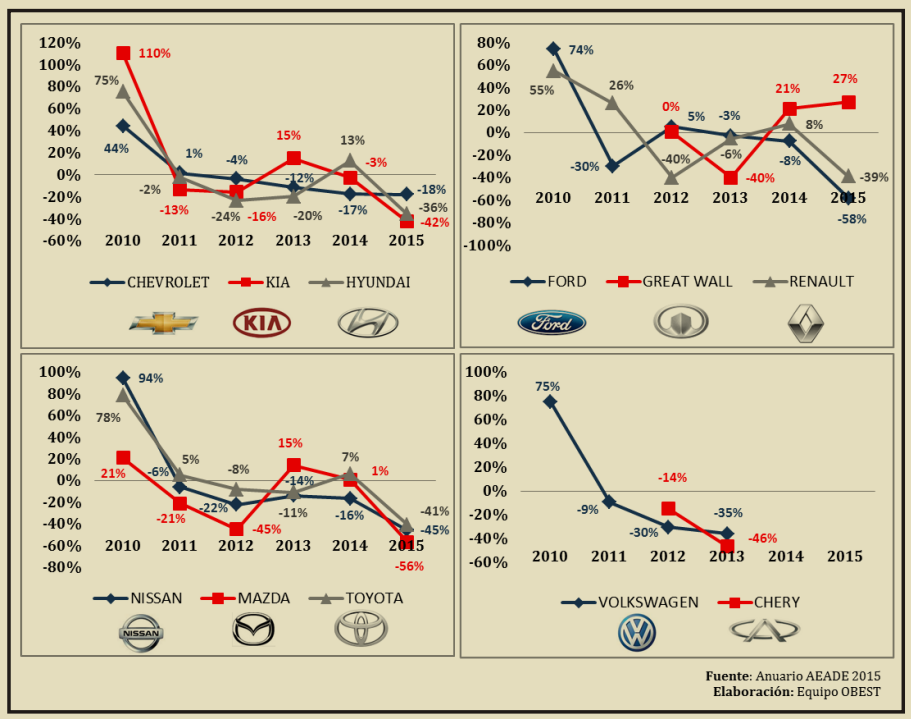

El $81 \%$ del parque automotor en la provincia de Tungurahua tiene entre 1 y 25 años con un importante 34\% en el rango de 1 a 5 años, con lo que, se puede concluir que gran parte de usuarios dispone de vehículos en buena condición relativamente por el tiempo de antigüedad. Se registran 5.495 unidades de menos de un año de antigüedad y 4.753 unidades con más de 30 años, esto representa el $4 \%$ para cada rango.

\section{Figura 5. Parque automotor en Tungurahua por años de antigüedad 2015}

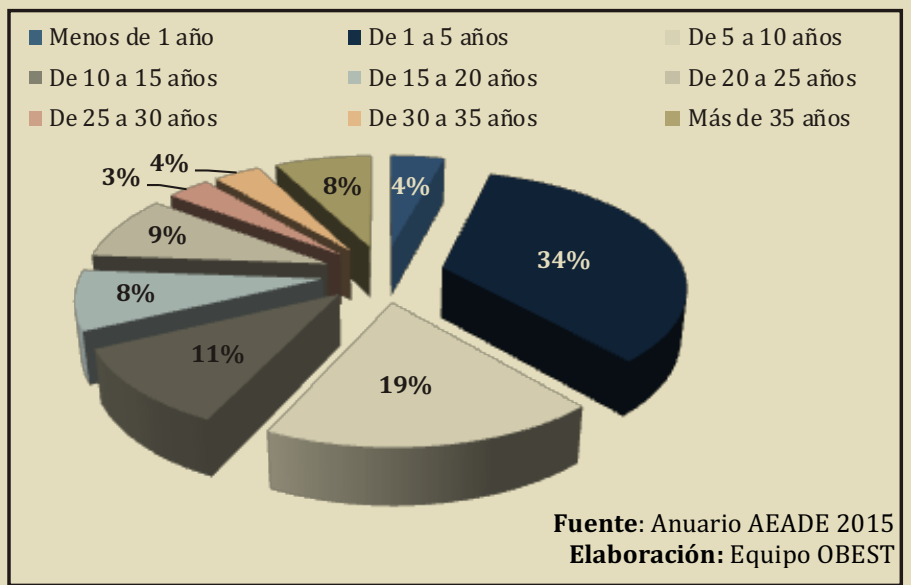

Durante el periodo 2010 - 2015 los precios a nivel nacional de camiones, SUV's, VAN's y automóviles muestran crecimiento constante con una notable diferencia en 2015, siendo el año en donde se han alcanzado los precios máximos de todo el periodo, a diferencia de buses y camionetas, segmentos que presentaron disminución en su precio. El segmento más vendido de automóviles registró un precio promedio de $\$ 18.342$ dólares, un precio máximo de \$23.965 dólares en 2015 y un precio mínimo de \$15.656 dólares en 2010. 
Figura 6. Precios promedio, máximo y mínimo por segmento Tungurahua 2010-2015

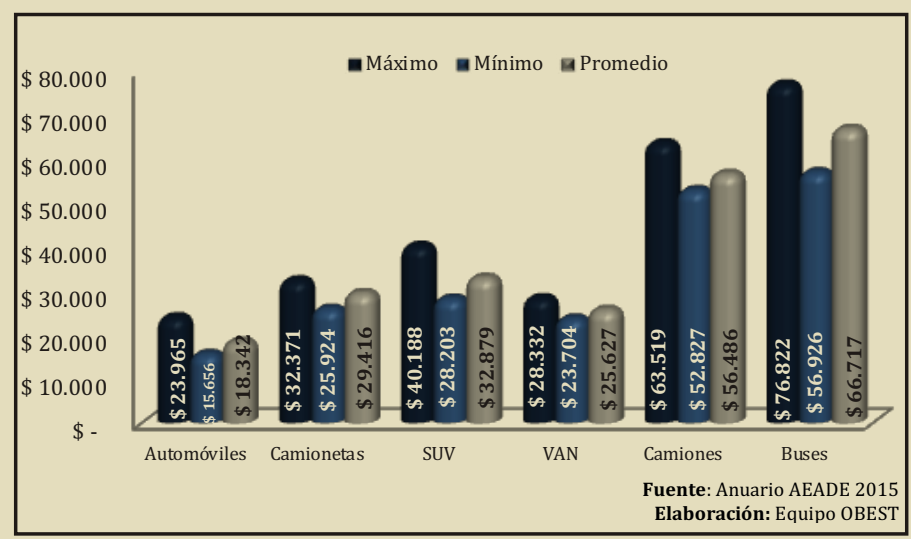

De la misma manera que se ha visto afectado el sector comercial automotor, se ha afectado el sector productor, es así que AYMESA, por ejemplo, ha disminuido su producción entre 2013 y 2015 en 7.465 unidades, OMNIBUS BB presenta incremento de producción de 2013 a 2014 pero en 2015 deja de producir 10.044 unidades, igual comportamiento tiene MARESA con disminución de $20 \%$ en 2014 y 5\% en 2015, CIAUTO es la única empresa que incrementa su producción en 59\% en 2014 y 53\% en 2015 lo que representa 1.061 unidades más de 2013 a 2015, es el mismo caso de la marca GREATWALL en venta de vehículos cuyo comportamiento aumentó por ser empresas nuevas en el mercado nacional.
Figura 7. Tasa de crecimiento de la producción nacional de vehículos 2013-2015

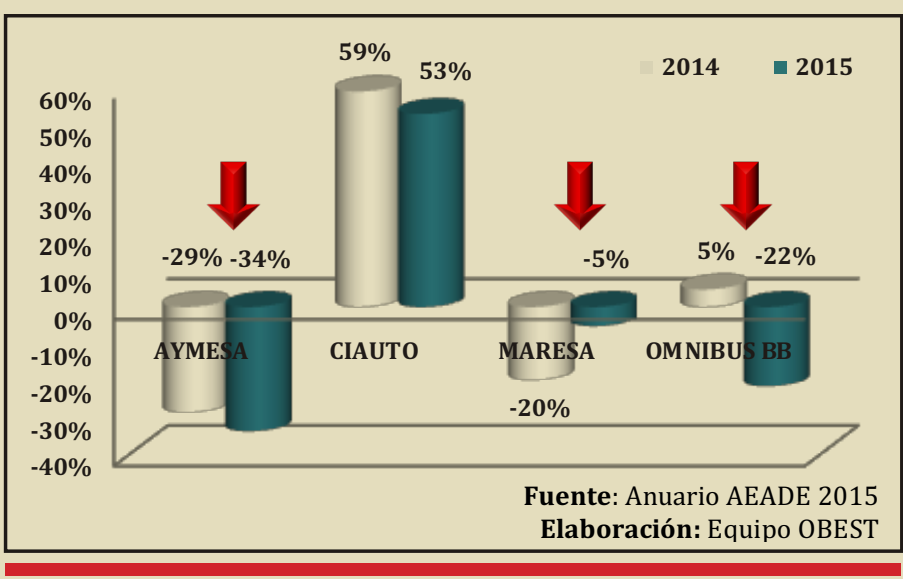

\section{Referencias:}

- Anuarios 2008, 2009, 2010, 2011, 2012, 2013, 2014 y 2015 AEADE Asociación de Empresas Automotrices del Ecuador, disponible en: https://sites.google.com/site/ aeadeecuador/sector-en-cifras

- Código Orgánico de la Producción, Comercio e Inversiones, Ecuador

- Primer Sondeo de percepción a comerciantes de vehículos usados realizado por e Observatorio Económico y Social de Tungurahua - OBEST. 2016

- Resoluciones 66-2012, 49-2014, 11 y 50-2015, 01-2016 COMEX Ecuador

- Revista GESTION, Ángela Meléndez Sánchez, El sector automotor, empeñado en su sobrevivencia, Pág. 32, Nro. 219 - 2013.

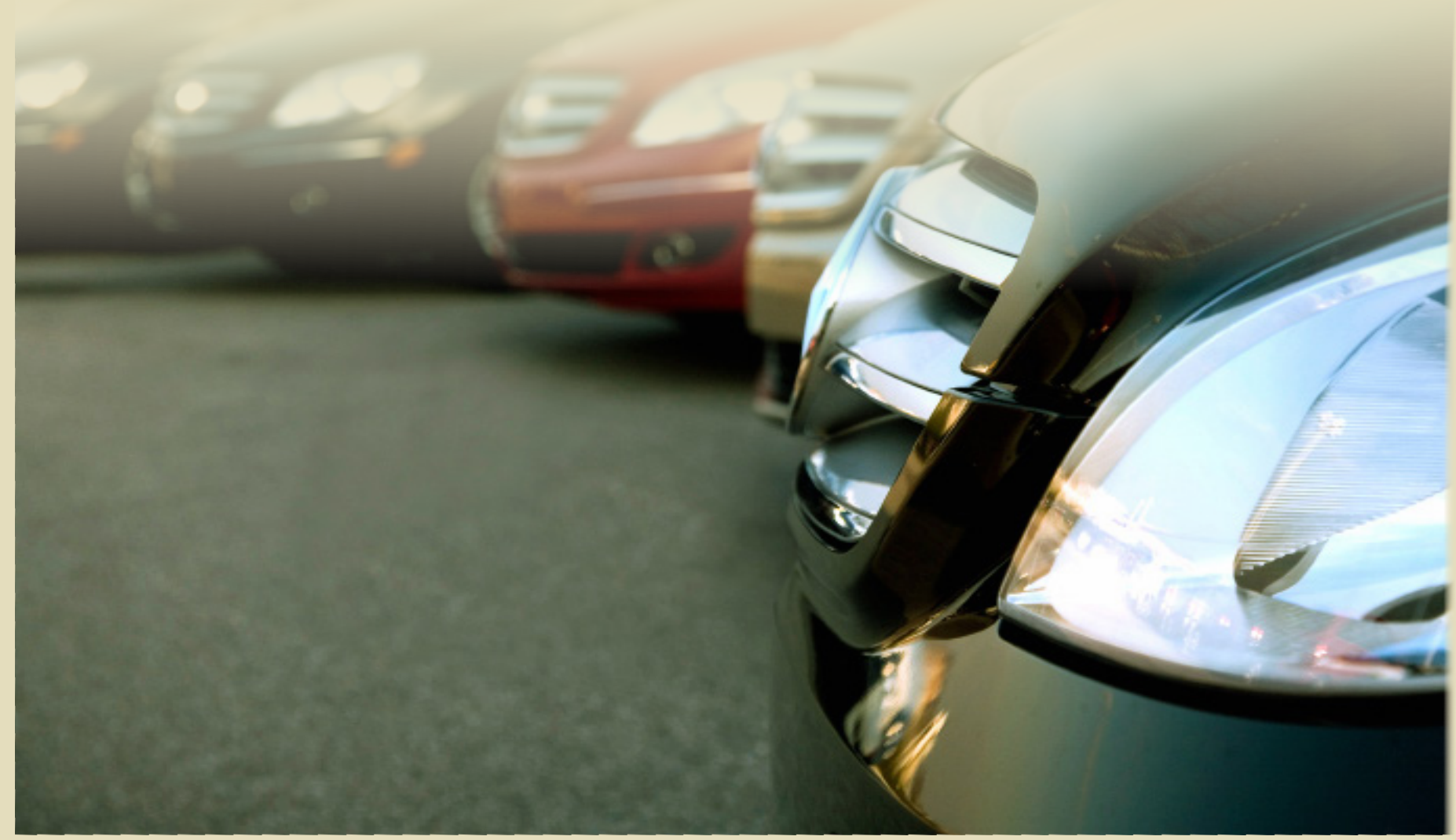

